\title{
Secondary postpartum haemorrhage with uterine artery pseudoaneurysm after caesarean section, managed with uterine artery embolisation
}

\author{
S Barahmeh, ${ }^{1} \mathrm{MD}(\mathrm{O} \& \mathrm{G})$; S H Edwan, ${ }^{2} \mathrm{MD}$ (Radiology) \\ ${ }^{1}$ Department of Obstetrics and Gynaecology, Consultative Arab Hospital, Ramallah, Palestine \\ ${ }^{2}$ Interventional Radiology Department, Consultative Arab Hospital, Ramallah, Palestine
}

Corresponding author: S Barahmeh (samersalman2@yahoo.com)

Uterine artery pseudoaneurysm (PA) is a rare but serious complication of caesarean section (C/S). If inadequately treated, it can lead to life-threatening postpartum haemorrhage. We report the case of a 28 -year-old woman who developed secondary postpartum haemorrhage resulting from uterine artery PA after C/S. Angiographic embolisation is a safe and effective procedure for treating postpartum haemorrhage resulting from PA in haemodynamically stable patients. However, uterine artery ligation may be the surgical procedure of choice for haemodynamically unstable patients when preservation of fertility is desired.

S Afr J Obstet Gynaecol 2017;23(1):28-30. DOI:10.7196/SAJOG.2017.v23i1.1105

Pseudoaneurysm (PA) of the uterine artery is an uncommon cause of delayed postpartum haemorrhage following cesarean section $(\mathrm{C} / \mathrm{S})$ or vaginal delivery. This condition may result in secondary postpartum haemorrhage, which is defined as haemorrhage that occurs between 24 hours, and 6 and 12 weeks postpartum. ${ }^{[1]}$ Although a diagnosis of retained gestational products or endometritis should be considered initially, a diagnosis of PA of the uterine artery and caesarean scar dehiscence should be considered after ruling out disseminated intravascular coagulation as a possible diagnosis when a patient presents with massive uterine bleeding without any associated symptoms, such as fever and tenderness, or subinvolution of the uterus. ${ }^{[2]}$

Haematoma formation involving the uterine artery is the main suggested mechanism associated with PA of the uterine artery. After haematoma formation, there is central liquefaction that leaves a cavity with turbulent blood flow as a result of persistent communication between the parent artery and the hematoma. A uterine artery PA develops when the uterine artery is lacerated or injured in a way that would breach the integrity of the three-layer arterial wall lining. While maintaining contact with the parent vessel, extravasating blood dissects through the tissues, finally establishing a connection with the uterine cavity, which causes a delayed haemorrhage. The boundaries of a false aneurysm are constituted by thrombus, as opposed to the three arterial layers as in a true aneurysm. Although Doppler ultrasound can aid in the assessment, uterine artery angiography is necessary to make the diagnosis and provides the subsequent means for embolisation. We present a case of a uterine artery PA presenting as delayed postpartum haemorrhage. ${ }^{[3]}$

\section{Case report}

A 28 -year-old para 3 was transferred to our institution at 27 days post operation with symptoms of excessive bleeding per vagina. She had undergone an elective low transverse segment $\mathrm{C} / \mathrm{S}$ delivery for breech presentation at a gestational age of 39 weeks at another hospital. Her intraoperative course was smooth and uneventful, and the estimated blood loss was $\sim 600 \mathrm{~mL}$. Her postoperative course was also uncomplicated until day 13 postoperatively.

On postoperative day 13 , she was readmitted to the same hospital for management of a delayed postpartum haemorrhage, which had developed abruptly with no previous history of abdominal pain, foul-smelling vaginal discharge or fever. She was hypotensive (blood pressure 80/45) and tachycardic (pulse of $110 \mathrm{bpm}$ ) with a haemoglobin of $6.8 \mathrm{~g} / \mathrm{dL}$. Her platelet count was 149 and her coagulation profile and biochemistry showed no derangements. A transabdominal ultrasound showed blood in the uterus, but there were no retained gestational products

After stabilisation with four units of cross-matched packed red blood cells, a dilatation and curettage was performed and tissue was sent to pathology for analysis. The bleeding stopped, and she was discharged on her second day in hospital, with a haemoglobin level of $10 \mathrm{~g} / \mathrm{dL}$. Pathology results showed no evidence of retained gestational products.

Two weeks later, she was admitted to the same hospital where she had had her C/S and surgical evacuation, with increased vaginal haemorrhage and severe lower abdominal pain. Although she was haemodynamically stable, with a BP of $110 / 65$ and a pulse of 88 bpm, her blood analysis revealed a haemoglobin level of $8.0 \mathrm{~g} / \mathrm{dL}$. Transvaginal sonography, with both colour and power Doppler modes, confirmed an empty uterus without signs of residual placental tissue in the uterine cavity. A hypoechoic lesion was detected in the isthmic region of her uterus and power Doppler imaging revealed blood flow within it.

Interventional radiology at our institution was consulted, as the patient desired future fertility. Pelvic angiography demonstrated a left uterine artery PA, which measured $4 \mathrm{~cm}$ in diameter, with extravasation of contrast into a pocket that connected to the uterine cavity.

After obtaining the patient's consent, selective left uterine artery embolisation was performed with a mixture of Gelfoam and contrast media, followed by one stainless steel coil insertion. The 


\section{CASE REPORT}

procedure was inititated by placing a number 4 French sheath into the right common femoral artery and passed retrograde into the right external iliac artery, right common iliac artery and aorta, respectively, at which point crossing over to the left common iliac artery, left internal iliac artery, and the left uterine artery. Selective catheterisation of the left uterine artery was carried out with a Cobra glide, Terumo Guidewire and Progreat microcatheter. Angiography showed a PA of the left uterine artery (Figs. 1 and 2), and embolisation was performed with Gelfoam and a stainless steel coil embolus. A post-embolisation angiography investigation was performed to ensure complete occlusion of the left uterine artery. A follow-up with colour Doppler ultrasound one day later showed that the aneurysmal cavity was filled with echogenic content and there was no evidence of blood flow within the cavity (Fig. 3).

\section{Discussion}

Postpartum haemorrhage remains one of the major causes of maternal mortality. Secondary postpartum haemorrhage is defined as excessive bleeding that starts any time from 24 hours after delivery up to 6 weeks postpartum, and most commonly occurs between 8 and 14 days postpartum. ${ }^{[1]}$ Common causes include retained gestational products, subinvolution of the placental bed, and endometritis. Rare causes include PA of the uterine artery, arteriovenous malformations, $\mathrm{C} / \mathrm{S}$ delivery and choriocarcinoma. When the more common causes have been excluded, pelvic angiography may be performed. PA of the uterine artery should be listed as a possible cause of postpartum haemorrhage after $\mathrm{C} / \mathrm{S}{ }^{[4]}$ Trauma to the uterine artery during surgery may cause a defect in the arterial wall, through which arterial blood escapes and diffuses to the adjacent tissues, resulting in the formation of a haematoma. When this haematoma is in continuity with the uterine artery, which supplies continuous blood flow, a PA forms. The absence of a three-layered arterial wall lining in a PA differentiates it from a true aneurysm. ${ }^{[5]}$

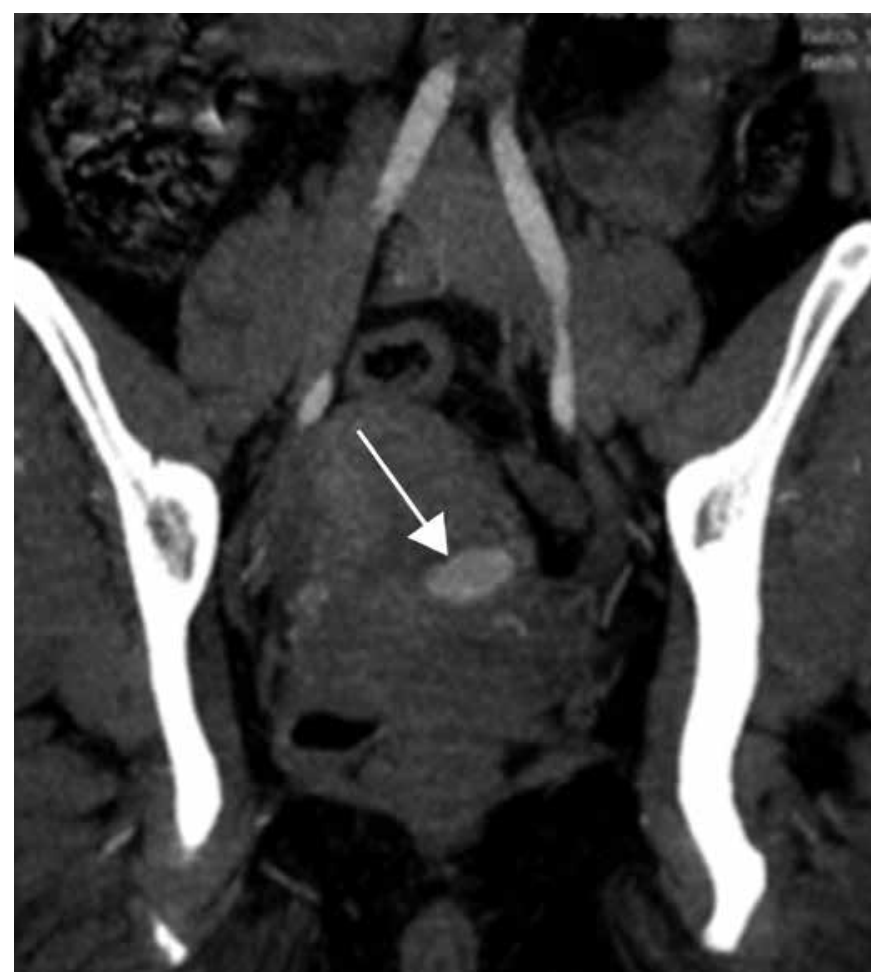

Fig. 1. Computed tomography angiography showing pseudoaneurysm of the left uterine artery, which measured $4 \mathrm{~cm}$ in diameter.

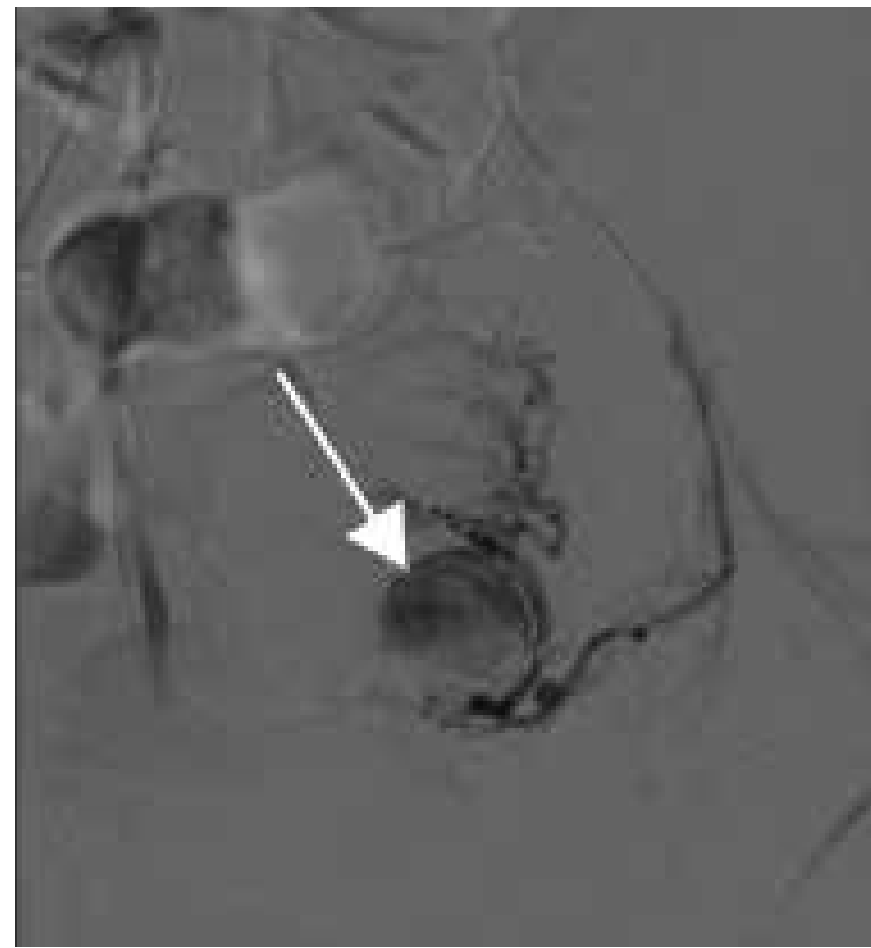

Fig. 2. Angiography showing pseudoaneurysm of the left uterine artery.

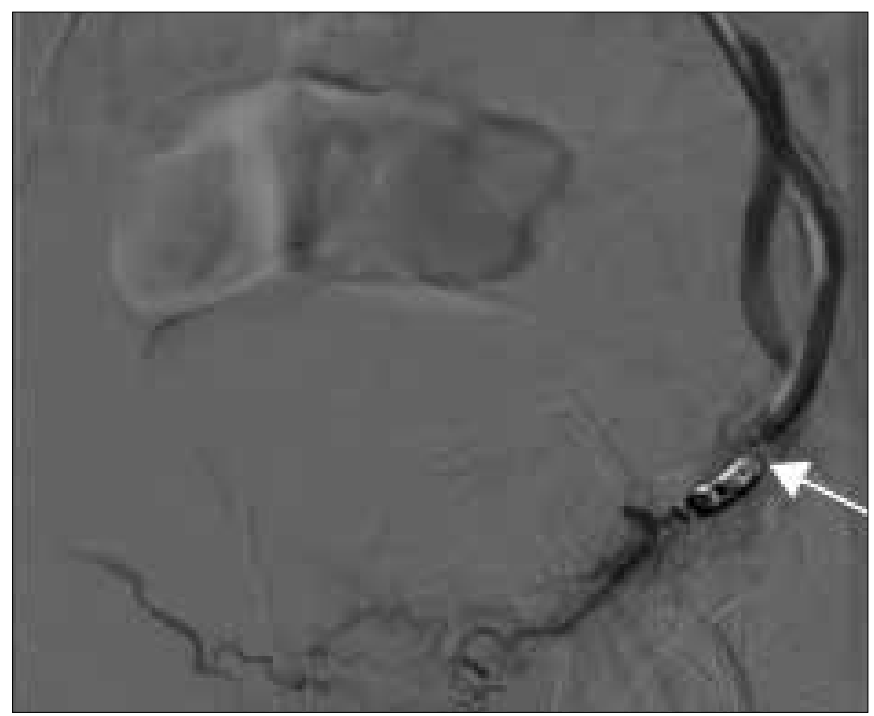

Fig. 3. Result after embolisation coil.

Pelage et al. ${ }^{[6]}$ evaluated the efficacy and safety of selective arterial embolisation of uterine arteries in women with delayed secondary postpartum haemorrhage. In their study $(N=14)$, pseudoaneurysms of the uterine artery were found in two participants and immediate resolution of external bleeding was observed after embolisation. In our study, there were no complications related to this invasive treatment. Other authors have described complications, including muscle pain and bladder necrosis. ${ }^{[1]}$ Our patient developed a PA $\sim 4$ weeks post-C/S delivery. Treatment was by angiographic embolisation of uterine arteries with Gelfoam and embolisation coils. In a retrospective review of eight women, Rosenthal and Colapinto $^{[7]}$ observed that angiographic arterial embolisation was the most useful clinical tool in the management of post-operative vaginal haemorrhage. 


\section{CASE REPORT}

Angiographic embolisation has many advantages when compared with hysterectomy including decreased morbidity, the ability to localise the bleeding site and provide a more distal occlusion than surgical ligation, and preservation of future fertility. Burchell ${ }^{[8]}$ demonstrated that bilateral internal iliac artery ligation was more effective than unilateral ligation in reducing the pulse pressure. It is possible that the redistribution and redirection of blood or hypoxia-induced neovascularisation allows bleeding to occur from the contralateral side after unilateral embolisation. Inadequate embolisation of a PA due to extrauterine feeding arteries, such as the internal pudendal artery, ovarian artery, inferior epigastric artery, or contralateral uterine artery can lead to embolisation failure. Hence, bilateral uterine embolisation is safe and more advantageous than unilateral embolisation. ${ }^{[3,9]}$

Uterine artery embolisation has become an effective and safe treatment for postpartum haemorrhage, allowing the preservation of reproductive function. Recent reports described the use of a thrombin injection directly into the pseudoaneurysm under ultrasound guidance, as a substitute for arterial embolisation; however, its indications and effectiveness have not yet been determined. The surgical approach may be more suitable in cases of acute and massive bleeding in which there is no time for embolisation, and may depend on the specific resources available in each institution. ${ }^{[2]}$

With newer modalities of treatment becoming available, hysterectomy for postpartum haemorrhage should be a last resort. Injuries to the vessel are more likely during a $\mathrm{C} / \mathrm{S}$, which could subsequently give rise to a PA. Doppler ultrasound and angiography are useful techniques to diagnose this condition. Selective embolisation of an affected vessel can be performed and bleeding can be arrested instantaneously. Being a minimally invasive procedure and posing limited risk to the mother, embolisation should be offered whenever feasible, and hysterectomy could be considered when the preservation of fertility is not important. On the other hand, uterine artery ligation and extirpation of uterine artery PA is another surgical choice for preserving fertility. ${ }^{[10]}$

Conflict of interest. The authors report no conflicts of interest.

1. Nanjundan P, Rohilla M, Raveendran A, Jain V, Khandelwal N. Pseudoaneurysm of uterine artery: A rare cause of secondary postpartum hemorrhage, managed with uterine artery embolisation. J Clin Imaging Sci 2011;1:14. https://doi.org/10.4103/2156-7514.76692

2. Yeniel AO, Ergenoglu AM, Akdemir A, Eminov E, Akercan F, Karadadaș N. Massive secondary postpartum hemorrhage with uterine artery pseudoaneurysm after cesarean section. Case Rep Obstet Gynecol 2013:2013:285846. https://doi.org/10.1155/2013/2858463

3. Sharma N, Ganesh D, Devi L, Srinivasan J, Ranga U. Prompt diagnosis and treatment of uterine arcuate artery pseudoaneurysm: A case report and review of literature. I Clin Diagnos Res 2013;7(10):2303-2306. https://doi.org/10.7860//CDR/2013/6063.3506

4. Zubor P, Kajo K, Dokus K, et al. Recurrent secondary postpartum hemorrhages due to placental site vessel subinvolution and local uterine tissue coagulopathy. BMC Pregnancy Childbirth 2014;14:80. https://doi.org/10.1186/1471-2393-14-80

5. Takeda A, Kato K, Mori M, Sakai K, Mitsui T, Nakamura H. Late massive uterine hemorrhage caused by ruptured uterine artery pseudoaneurysm after laparoscopic-assisted myomectomy. Minim Invasive Gynecol 2008,15(2):212-216. https://doi.org/10.1016/j.jmig.2007.09.006

6. Pelage JP, Soyer P, Repiquet D, et al. Secondary postpartum hemorrhage: Treatment with (elective arterial embolisation. Radiology 1999:12:385-389. https//doi.org/10.1148/ radiology.212.2.r99j105385

7. Rosenthal DM, Colapinto R. Angiographic arterial embolization in the management of postoperative vaginal hemorrhage. Am J Obstet Gynecol 1985;151(2):227-231. https://doi.org/10.1016/0002

8. Burchell RC. Internal iliac artery ligation: Hemodynamics. Obstet Gynecol 1964;24:737-739.

9. Cooper BC, Hocking-Brown M, Sorosky JI, et al. Pseudoaneurysm of the uterine artery requiring bilateral uterine artery embolization. J Perinatol 2004;24(9):560-562. https://doi.org/10.1038 sj.jp.7211119

10. Chitra TV, Panicker S. Pseudoaneurysm of uterine artery: A rare cause of secondary postpartum hemorrhage. J Obstet Gynaecol India 2011;61(6):641-644. https://doi.org/10.1007/s13224-011$0096-6$ 